\title{
Long-term stability of $0.1 \%$ rapamycin hydrophilic gel in the treatment of facial angiofibromas
}

\author{
Guillaume Le Guyader, ${ }^{1}$ Victoire Vieillard, ${ }^{1}$ Karine Andrieux, ${ }^{2}$ Mylène Rollo, ${ }^{1}$ \\ Olivier Thirion, ${ }^{1}$ Pierre Wolkenstein, ${ }^{3}$ Muriel Paul ${ }^{1}$
}

${ }^{1}$ Pharmacy Department, Henri Mondor Hospital Group, AP-HP, Creteil, France

${ }^{2}$ Unité de Technologies Chimiques et Biologiques pour la Santé (UTCBS), UMR CNRS 8258 U1022 INSERM, Université Paris Descartes, Paris, France

${ }^{3}$ Department of Dermatology, Henri Mondor Hospital Group, AP-HP, Créteil, France

\section{Correspondence to} Dr. Guillaume Le Guyader, Pharmacy Department, Henri Mondor Hospital Group, Créteil 94010, France; leguyader. guillaume@gmail.com

Received 25 July 2018 Revised 4 October 2018 Accepted 9 October 2018 Published Online First 10 November 2018

EAHP Statement 3: Production and Compounding.

\section{ABSTRACT}

Objectives In recent years, various formulations containing rapamycin, mainly petrolatum-based, have been tested on facial angiofibromas in tuberous sclerosis. They are often poorly tolerated due to irritation and bleeding. In addition, their effectiveness was insufficient in young adults. The objective of this study was to develop and characterise a hydro-alcoholic gel containing solubilised rapamycin. The stability of the product stored at $4^{\circ} \mathrm{C}$ was evaluated over 1 year.

Methods Two different $0.1 \%$ rapamycin gels were formulated with or without $\alpha$-tocopherol and urea. Different methods were used to characterise the gels: HPLC, gas chromatography, pH, visual observation and optical microscopy. A physico-chemical and microbiological stability study was also conducted for 1 year at $4^{\circ} \mathrm{C}$.

Results Gels were physically and microbiologically stable after 1 year at $4^{\circ} \mathrm{C}$ : organoleptic characteristics and $\mathrm{pH}$ unchanged, no significant decrease in rapamycin was observed, tocopherol droplet size was constant and rheological behaviour was not altered.

Conclusions This study describes a new gel formulation to improve skin penetration using various excipients to promote skin tolerance. This study provides, for the first time, detailed stability data for a hydro-alcoholic rapamycin gel.

\section{INTRODUCTION}

Tuberous sclerosis complex (TSC) is an autosomal dominant disease, characterised by the appearance of multiple hamartomas. Facial angiofibromas are one of the most common skin manifestations. They can be very disfiguring and can have a significant impact on patients' quality of life. ${ }^{1}$ They result from the overactivation of the mammalian target of rapamycin (mTOR) in dermal cells, which stimulates the proliferation of fibroblasts and keratinocytes and angiogenesis. $^{23}$

Many symptomatic treatments have been developed to reduce the occurrence of angiofibromas (eg, cryosurgery, laser ablation...). But some of them are costly and the results are disappointing, with frequent recurrence and should require regular sessions. ${ }^{45}$ Recently, based on the pathophysiology of the disease, the interest of mTOR inhibitors such as rapamycin or everolimus has been studied. In order to limit systemic side effects due to oral administration, different topical forms have been prepared with these drugs. Rapamycin has been the most studied mTOR inhibitor in the past 10 years, used as an oral solution or as an ointment with crushed petrolatum tablets on the skin. Regardless of blood concentration, efficacy and safety have been demonstrated. ${ }^{67}$ However, Rapamune oral solution was not suitable for topical administration in terms of tolerance, causing irritation and burning sensations, probably due to high surfactant levels. ${ }^{8}$ Moreover, rapamycin being insoluble in petrolatum or Dexeryl, the cutaneous bioavailability of these preparations should be low, which explains a certain delay in reaching a clinical response and the lack of total disappearance in adults with severe angiofibromas. For children, a better response was observed probably due to their skin characteristics, i.e. thinner and more permeable. ${ }^{9}$ With regard to ointment with tablets crushed in petrolatum, the presence of solid fragments may be responsible for the risk of bleeding and an uncomfortable sensation of oily skin has been observed. Recently, Bouguéon et al proposed a cream with $0.1 \%$ rapamycin solubilised with 5\% Transcutol to avoid any bleeding problem and this oily sensation, with stability data. ${ }^{10}$

Consequently, this work aimed to develop and characterise a hydro-alcoholic gel containing rapamycin solubilised and surfactant-free to improve the bioavailability of the active molecule, obtain a good appearance and allow skin tolerance. Finally, a long-term stability study at $4^{\circ} \mathrm{C}$ was performed to determine its shelf life.

\section{Methods}

\section{Gel preparation}

Two formulations were developed (table 1). Formula 1 (F1) was formulated without urea and $\alpha$-tocopherol contrary to Formula 2 (F2). Carbopol-974P (Duchefa-Farma, Nederland) was used as a gelling agent. Rapamycin (Inresa, France) was solubilised in ethanol (VWR, France). Then, glycerol was added with or without $\alpha$-tocopherol and urea at room temperature (Sigma-Aldrich, France). Twenty grams of gel were then packaged in aluminium tubes (Cooper, France) and stored in a controlled cold area $\left(5^{\circ} \mathrm{C} \pm 3^{\circ} \mathrm{C}\right)$ for the duration of the study.

\section{Chromatographic conditions}

The method, based on the publication of Il'ichev et $\mathrm{al}^{11}{ }^{11}$ was validated according to the International Consensus on Harmonisation guidelines (ICHQ2R1). Samples were analysed using an Ultimate 3000 coupled with a Photodiode Array Detector PDA-3000 (Thermo-Fisher-Scientific, France) operating between 190 to $800 \mathrm{~nm}$. Separation was achieved using an Interchim $250 \times 4.6 \mathrm{~mm}$ KR-C18 $(5 \mu \mathrm{m})$ column maintained at $40^{\circ} \mathrm{C}$. The mobile phase A consisted of a mixture of 


\begin{tabular}{|c|c|c|}
\hline Component & F1 & F2 \\
\hline Rapamycin & $0.02 \mathrm{~g}$ & $0.02 \mathrm{~g}$ \\
\hline Carbopol-974P & $0.15 \mathrm{~g}$ & $0.15 \mathrm{~g}$ \\
\hline Glycerol & $3.05 \mathrm{~g}$ & $3.05 \mathrm{~g}$ \\
\hline Absolute ethanol & $6 \mathrm{~g}$ & $6 \mathrm{~g}$ \\
\hline$\alpha$-tocophérol & I & $0.04 \mathrm{~g}$ \\
\hline Urea & 1 & $0.2 \mathrm{~g}$ \\
\hline Sterile water & QS $20 \mathrm{~g}$ & QS $20 \mathrm{~g}$ \\
\hline
\end{tabular}

water-formic acid 0.003 vol\% (Merck, Germany) and mobile phase B was acetonitrile-formic acid 0.003 vol\%. Acetonitrile and methanol (Chromasolv) were HPLC-grade (Sigma-Aldrich, France). The gradient programme used was an elution gradient of 50:50 to 10:90 (phase A:phase B) over $25 \mathrm{~min}$. The flow rate was $1.0 \mathrm{~mL} / \mathrm{min}$ and the injection volume was $50 \mu \mathrm{L}$. Rapamycin detection was processed at $277 \mathrm{~nm}$.

\section{Rapamycin extraction and recovery}

To appreciate the extraction coefficient, five different rapamycin concentrations in gel were prepared $(0.06,0.08,0.1,0.12 \%$ and $0.14 \%$ ) and measured by HPLC. For this, $0.1 \mathrm{~g}$ of the preparation was weighed and poured in a $10 \mathrm{~mL}$ volumetric flask. Methanol was added and the flask was placed in an ultrasonic bath for $10 \mathrm{~min}$. The resulting solution was centrifuged in a Sigma centrifuge $1-14 \mathrm{~K}$ at $10000 \mathrm{rpm}$ for $5 \mathrm{~min}$ and the supernatant was removed and analysed by HPLC. This solution was compared with rapamycin methanol stock solution at the same concentration.

\section{Forced degradation}

Stress studies were performed to demonstrate specificity of the stability-indicating method. To identify one major degradant of rapamycin, that is, secorapamycin, a standard solution at $10 \mu \mathrm{g} /$ $\mathrm{mL}$ of secorapamycin (Cayman Chemical, USA) diluted in methanol, was subjected to the HPLC method.

For each formulation, $0.1 \mathrm{~g}$ of gel $(\mathrm{n}=3)$ was subjected to a stress condition of acid $\left(0.1 \mathrm{n} \mathrm{HCl}\right.$ : heated for 1 hour at $\left.40^{\circ} \mathrm{C}\right)$, base $\left(0.01 \mathrm{n} \mathrm{NaOH}\right.$ : heated for 1 hour at $\left.40^{\circ} \mathrm{C}\right)$, and oxidation $\left(\mathrm{H}_{2} \mathrm{O}_{2}\right.$ 1vol: heated at $40^{\circ} \mathrm{C}$ for $1,2,3,6$ and 24 hours, white light). Then, an extraction was performed under the same conditions as presented above and analysed by HPLC. Blank gel was also tested alone.

\section{Organoleptic characteristics/pH}

The different organoleptic characteristics evaluated were:

- Appearance: An aliquot of gel was placed on a microscope slide and was inspected visually for their colour, homogeneity (presence of any aggregates) and grittiness (presence of particles or grits).

- Consistency: The stickiness was evaluated by spreading an aliquot of blank gel (without rapamycin) on the skin.

- Size and repartition of $\alpha$-tocopherol droplets and detection of possible rapamycin crystals: Microscopic observation (OlympusIM coupled to a Sony camera XCD-U100CR) was performed to ensure the homogeneity of the gel by searching the absence of aggregates and following the evolution of the size of tocopherol droplets. This analysis was performed on about 100 units for each formula. The diameter, width and surface of lipid droplets were evaluated.
- pH-determination: it was determined before each analysis using a calibrated $\mathrm{pH}$-meter Consort-P901.

Determination of residual ethanol by gas chromatography

Residual ethanol was analysed using Ultra Trace GC gas chromatography system (Thermo Electron, Italy). The column was an Interchim $30 \mathrm{~m} \times 0.53 \mathrm{~mm}$ UB-624 $(3 \mu \mathrm{m})$ column maintained at $40^{\circ} \mathrm{C}$ for $10 \mathrm{~min}$. The flow rate of carrier gas is $6 \mathrm{~mL} / \mathrm{min}$. A solution of acetonitrile $1 \mathrm{~g} / \mathrm{L}(\mathrm{MeCN})$ was used as the internal standard. For the determination of residual ethanol, an aliquot of gel $(50 \mathrm{mg})$ was weighed and poured with water and diluted in a $20 \mathrm{~mL}$ volumetric flask. Then, $1 \mu \mathrm{L}$ of 50:50 (Sample:MeCN) was injected. The residual ethanol was expressed in percentage $(\mathrm{g} / 100 \mathrm{~mL})$.

\section{Rheological measurements}

Rheological measurements were performed on an Anton Paar MCR-102 (Modular Compact Rheometer, Austria) equipped with plate-plate geometry of $100 \mathrm{~mm}$ diameter and Peltier temperature control device with thermostatic hood. Viscosity was measured as a function of the shear rate for $\mathrm{d} \gamma / \mathrm{dt}$ between 0.1 and $100 \mathrm{~s}^{-1}$ at $20.0^{\circ} \mathrm{C} \pm 0.1^{\circ} \mathrm{C}$.

\section{Stability study}

For the stability study, five batches were prepared for $\mathrm{F} 1(\mathrm{n}=5)$ and four batches for F2 $(n=4)$, and duplicate samples were analysed daily for each batch.

Samples $(0.5 \mathrm{~g})$ were withdrawn from tubes stored at $5^{\circ} \mathrm{C} \pm 3^{\circ} \mathrm{C}$, at different times (day): 0, 2, 7, 14, 30, 65, 100 and 365 .

Different methods have been used to characterise both formulations, evaluate their microbial quality and study the drug physicochemical stability. For each time, the organoleptic characteristics and $\mathrm{pH}$ were evaluated, the percentage of rapamycin remaining and the residual ethanol concentration. The rheological properties were evaluated at day 0,30 and 365 , and the microbiological cleanliness was evaluated at day 0 and day 365 .

The expiry date (days), based on a limit of $5 \%$ degradation, was calculated from the determination of T95\% as part of the process of assigning product shelf life in accordance with the recommendations of $\mathrm{ICH}$.

\section{Microbiological stability}

Microbial contamination of formulations has been tested in accordance with the European Pharmacopoeia. 1g sample to be tested, freshly manufactured or already stored for 1 year at $4^{\circ} \mathrm{C}$ were diluted to $1 / 50$ in meat peptone neutral solution. $5 \mathrm{~mL}$ of solution was filtered at a rate of 1 filter $/ \mathrm{ml}$. Thereafter, the filters were successively rinsed with $50 \mathrm{~mL}$ sodium chloride solution and with $50 \mathrm{~mL}$ meat peptone neutral solution to remove residual ethanol.

After contact of a filter membrane with soybean casein digest agar, the plates were incubated at $32^{\circ} \mathrm{C}$ and total aerobic microbial count (TAMC) was determined after 3-day storage. The use of Sabouraud dextrose agar incubated at $25^{\circ} \mathrm{C}$ for 5 days determined the total combined yeasts/moulds count (TYMC). Results were expressed as a colony forming unit per gram $(\mathrm{CFU} / \mathrm{g})$ against specification limits $\left(<10^{2} \mathrm{CFU} / \mathrm{g}\right.$ for TAMC and $<10 \mathrm{CFU} / \mathrm{g}$ for TYMC).

\section{Statistical analysis}

All values were expressed as mean \pm SD deviation. Data were compared using one-way ANOVA. In all cases, a difference was considered significant at $\mathrm{P}<0.05$. 


\begin{tabular}{lll}
\hline $\begin{array}{l}\text { Table } 2 \\
\text { formulation }\end{array}$ & Organoleptic characteristics and pH values for each gel \\
\hline Organoleptic characteristics & F1 & F2 \\
\hline Appearance and stickiness & $\begin{array}{l}\text { Smooth, homogeneous } \\
\text { and no sticky }\end{array}$ & $\begin{array}{l}\text { Smooth, homogeneous } \\
\text { and no sticky }\end{array}$ \\
\hline Odour & Slight alcohol odour & Slight alcohol odour \\
Rapamycin crystals & Not observed & Not observed \\
Colour & Transparent & Slightly opalescent \\
Size of $\alpha$-tocophérol drops & Not applicable & $8.7 \pm 3.8 \mu \mathrm{m}$ \\
\hline pH value & $6.6 \pm 0.1$ & $6.7 \pm 0.1$ \\
\hline
\end{tabular}

\section{RESULTS}

\section{Characterisation of the formulations}

Physical and chemical characteristics of rapamycin gels

The organoleptic characteristics and $\mathrm{pH}$ of the different gels are summarised in table 2 . Both formulations were smooth, homogeneous with a slight alcohol odour and a $\mathrm{pH}$ was compatible with skin application. A slight opalescence was observed in F2 due to tocopherol droplets. In addition, microscopic observations revealed the absence of rapamycin crystals in both formulations, confirming the dissolution of the drug in these gels. Both formulations had a rheofluidifying character. The viscosity of the gels is close to 20 Pa.s, completely compatible with a cutaneous application.

\section{Determination of residual ethanol by gas chromatography}

The GC chromatogram showed that ethanol and acetonitrile were eluted at $2.4 \mathrm{~min}$ and $2.9 \mathrm{~min}$, respectively. The ethanol concentrations were $18.5 \% \pm 1.5 \%$ for $\mathrm{F} 1$ and $18.7 \% \pm 2.8 \%$ for F2, respectively.

\section{Rapamycin content in gels}

Validation of the HPLC method to measure rapamycin content in gel This method permitted the obtainment of a well-defined main peak (asymmetry factor $=1$ ) and a good resolution to ensure a good separation between isomers. As shown in figure 1, the retention times were $20 \mathrm{~min}$ and $21 \mathrm{~min}$ for the rapamycin beta $(\beta)$ and gamma $(\gamma)$ isomer, respectively. Linear response signal versus concentration was obtained $\left(\mathrm{r}^{2}=0.998\right)$. The mean recovery rate was $100.03 \% \pm 1.3 \%$.

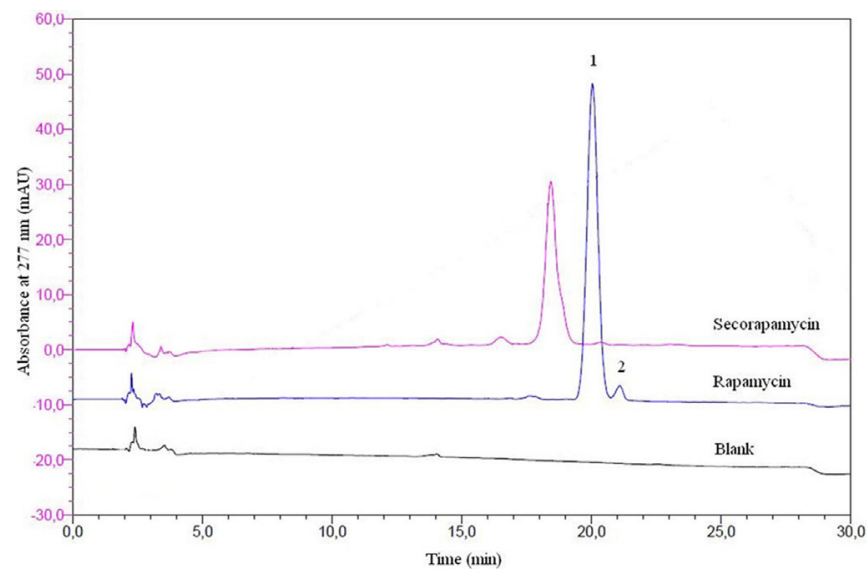

Figure 1 Typical overlay RP-HPLC chromatograms of blank gel (black line), rapamycin gel (blue line) isomers $\beta(1)$ and $\gamma(2)$ after methanol extraction. Standard solution at $10 \mu \mathrm{g} / \mathrm{mL}$ of secorapamycin in methanol (pink line). Column: $5 \mu \mathrm{m}$, Interchim C18 $250 \times 4.6 \mathrm{~mm}$; temperature $40^{\circ} \mathrm{C}$; injection volume $50.0 \mu \mathrm{L}$; and UV detection $277 \mathrm{~nm}$. Mobile phase: water/ formic acid 0.003 vol\% and acetonitrile/formic acid 0.003 vol\%.

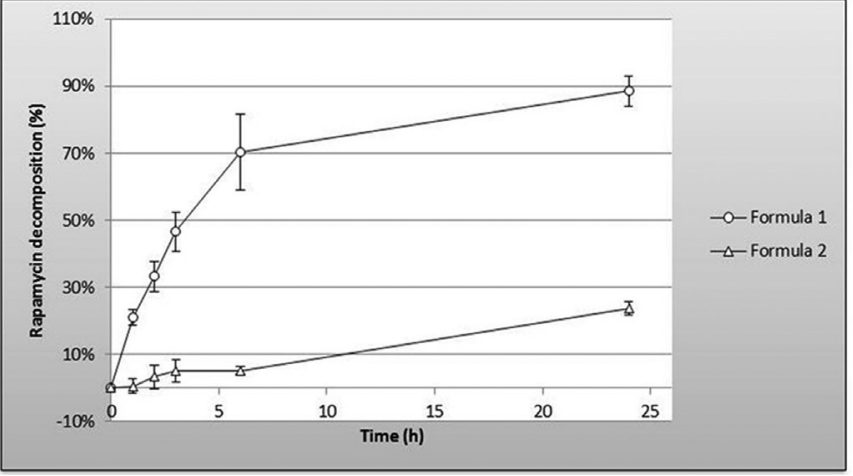

Figure 2 Oxidative stress of gel formations analysed by RP-HPLC. Stressed conditions: $\mathrm{H}_{2} \mathrm{O}_{2} 1$ vol; heated at $40^{\circ} \mathrm{C}$ for $1,2,3,6$ and 24 hours, white light. The addition of tocopherol in Formula 2protects rapamycin from oxidative stress.

The relative SD were $1.3 \%$ and $2.0 \%$ for the repeatability and the intermediate precision, respectively.

\section{Rapamycin extraction and recovery}

The extraction in methanol from the gel, allowed a recovery of $92.2 \% \pm 0.1 \%$ for $\mathrm{F} 1$ and $94.6 \% \pm 0.1 \%$ for F2. The drug content in gels was $0.098 \% \pm 0.003 \%$ for F1 and $0.090 \% \pm 0.005 \%$ for F2.

\section{Forced degradation}

No interfering peak was observed regardless of the stressed condition applied. Adequate resolution between the peaks relevant to rapamycin and rapamycin degradation products was also observed. Moreover, the addition of tocopherol in F2 protects rapamycin against oxidative stress: \%rapamycin decomposition at 24 hours was $88.6 \% \pm 4.4 \%$ versus $23.6 \% \pm 2.0 \%$ for F1 and $\mathrm{F} 2$, respectively (figure 2 ).

\section{Stability study}

\section{Physicochemical characteristics}

The organoleptic characteristics (appearance, modification of colour, stickiness) of the gels remained unchanged throughout the period tested. No particle of rapamycin was detected by microscopic examination. Moreover, no modification of rheological character of gels was observed as a function of time (figure 3).

The $\alpha$-tocopherol droplets size did not significantly change throughout the storage period $(8.2 \pm 5.3 \mu \mathrm{m}, \mathrm{P}=0.09)$.

Regarding $\mathrm{pH}$, no significant change after 1 year of storage was observed, $\mathrm{pH}$ were $6.7 \pm 0.1(\mathrm{P}=0.13)$ for $\mathrm{F} 1(\mathrm{n}=3)$ and $6.7 \pm 0.08(P=0.71)$ for $F 2(n=3)$.

\section{Determination of residual ethanol by gas chromatography}

Throughout the storage period, residual ethanol did not significantly alter $(\mathrm{P}=0.92$ and $\mathrm{P}=0.40$ for $\mathrm{F} 1$ and $\mathrm{F} 2$, respectively). The solvent was within $20.0 \% \pm 2.2 \%$ for F1 $(n=5)$ and $20.5 \% \pm 2.5 \%$ for F2 $(n=4)$ as originally found. From the beginning of the study, a $33 \%$ loss was observed with respect to the theoretical concentration (ie, 30\% weight/weight).

\section{Percentage of rapamycin remaining}

The evolution of the remaining percentage of rapamycin is indicated in table 3 . No significant change occurred after 1 year storage $(\mathrm{P}=0.17$ for $\mathrm{F} 1$ and $\mathrm{P}=0.12$ for $\mathrm{F} 2)$. The mean concentration levels were $99.4 \% \pm 2.2 \%$ and $101.0 \% \pm 1.9 \%$ for $\mathrm{F} 1(\mathrm{n}=5)$ and F2 $(\mathrm{n}=4)$, respectively. Due to these results, the 


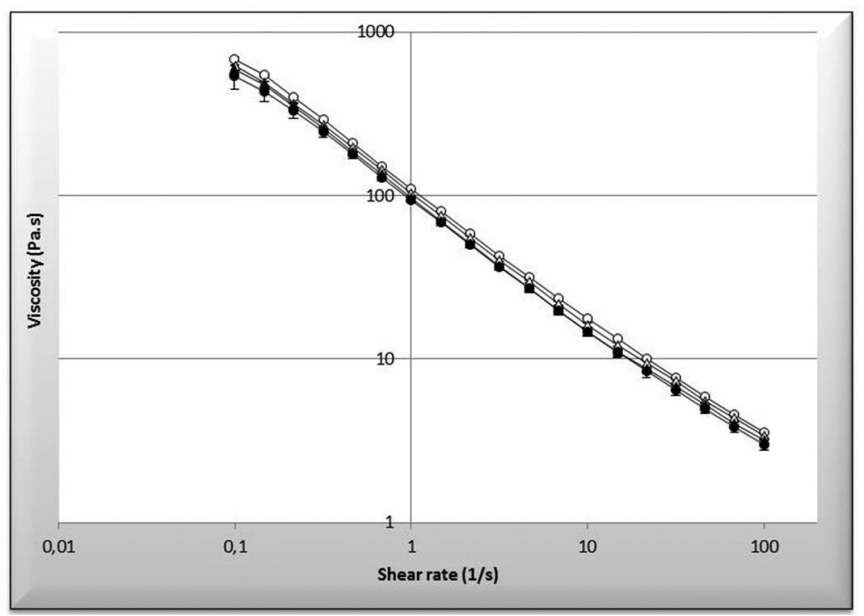

Figure 3 Rheological measurement over time of the gel. Empty round corresponds to Formula 1 at day 0 versus full round corresponds to Formula 1 at 360 days. Empty triangle corresponds to Formula 2 at day 0 versus full triangle corresponds to Formula 2 at 360 days.

T95\% was not calculated because there was no evidence that the gels can be stored more than 1 year without any loss of drug. As shown in figure 4, both chromatograms at days 0 and 365 for F2 were comparable. The same result was observed with F1. Moreover, the degradation products, notably secorapamycin, were undetected.

\section{Microbiological stability}

All preparations conformed to the current European Pharmacopoeia 8.0 and showed that the TAMC and the TYMC were less than $10 \mathrm{UFC} / \mathrm{g}$ in the storage conditions at $4^{\circ} \mathrm{C}$. In addition, there were no suspected colonies of Staphylococcus aureus and Pseudomonas aeruginosa found in the selective media of any samples. Moreover, the same result was observed after 1 year of storage.

\section{DISCUSSION}

The results showed that the hydro-alcoholic gel containing $0.1 \%$ rapamycin and formulated with or without $\alpha$-tocopherol and urea, was stable for at least 1 year at $+4^{\circ} \mathrm{C}$ and protected from light. No degradation products were detected and there were no changes in the physico-chemical or microbiological characteristics of the gel. The gel had a smooth, homogeneous texture with no trace of rapamycin precipitate. In addition, the residual ethanol content remained stable for 1 year. Nevertheless, a loss of 33\% ethanol after manufacture compared with the theoretical concentration was observed. This loss is probably due to the manufacturing process since the stability study revealed a constant percentage. This loss could have resulted in rapamycin precipitation, but microscopic analysis of the gel did not detect undissolved rapamycin particles. In F2, the droplet size distribution of $\alpha$-tocopherol remained unchanged after 1 year, which implies good physical stability.

Among the different types of topical preparations based on rapamycin, a single cream containing $0.1 \%$ rapamycin solubilised in Transcutol and mixed with a marketed cream (Excipial) was studied for its stability. ${ }^{10}$ However, stability has only been achieved over

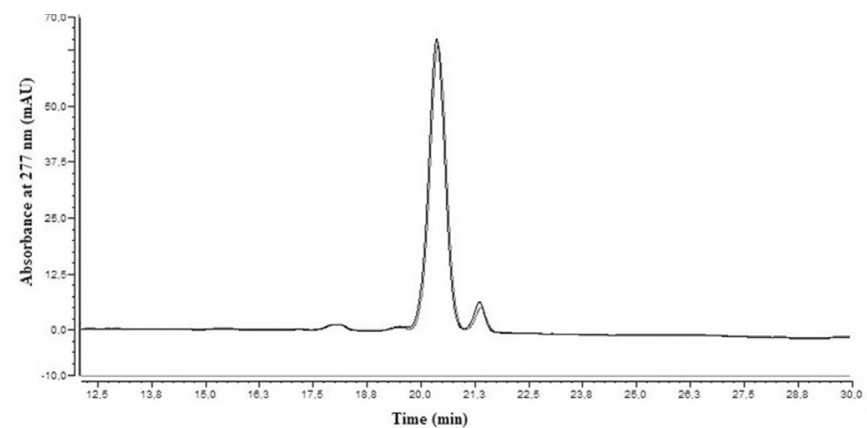

Figure 4 Chromatogram of rapamycin gel at D0 versus D365 (Formula 2). Both peaks were surimposable, which reflected the stability of the product over the time in the preparation. The same result was observed with Formula 1.

85 days while our gel is stable at $4^{\circ} \mathrm{C}$ for a shelf life of at least 1 year. Moreover, our results were obtained using a highly selective and validated RP-HPLC method, allowing good separation of isomers and degradation products, including secorapamycin, an open ring isomer with extremely low immunosuppressive activity. ${ }^{11}$ In addition, it has been shown that this method indicates stability with a very good extraction coefficient (more than 90\% for both formulas in hydro-alcoholic gel compared with $64.2 \% \pm 1.2 \%$ in Excipial). ${ }^{10}$

The choice to develop a $0.1 \%$ rapamycin hydrogel was based on the literature. As shown by several studies, the efficacy and tolerance of the different preparations used are more or less good, depending on the concentration of rapamycin, the type of raw material (API standard powders, ground tablets or oral solutions of rapamycin), the vehicle used for the topical route and the age of the patients. ${ }^{6891213}$

It should be noted that the use of crushed tablets or rapamycin powder, dispersed in a fatty base such as petrolatum or Dexeryl, would limit skin bioavailability because the drug is not fully solubilised in the topical formulation. Therefore, most authors used concentrations up to $0.1 \%$, particularly $0.2 \%$ and $1 \%$, or increased the frequency of application (twice daily). ${ }^{671415}$ In a prospective study, Malissen et al showed a significant improvement in FASI by using 1\% Dexeryl rapamycin cream, resulting in complete clearance of $50 \%$ at 9 months, with no further benefit. ${ }^{15}$ In addition, it was assumed that the bleeding was due to incomplete crushing of the tablets. The oral solution was also very irritating, probably due to the high level of surfactant, and made the skin oily, leading to poor compliance. ${ }^{12} 16$

So, in order to limit tolerance problems and to increase skin bioavailability, rapamycin solubilisation has been identified as a key element of the process. Ethanol was chosen as the solubilising solvent. It is well known that ethanol is capable of improving the cutaneous penetration of drugs by increasing the porosity of the stratum corneum. ${ }^{17}$ To increase patient comfort, we have added some excipients to our formulation. Glycerol is a hygroscopic substance used as a humectant and emollient agent to promote skin hydration. ${ }^{18}$ In addition, topical application of glycerol improves skin elasticity and helps repair the epidermal barrier. ${ }^{19}$ The addition of $\alpha$-tocopherol, an effective antioxidant, has been used to protect rapamycin from oxidation and also to

Table 3 Remaining percentage of rapamycin in preparations over the time

\begin{tabular}{lllllllll}
\hline Time (days) & $\mathbf{0}$ & $\mathbf{2}$ & $\mathbf{7}$ & 14 & 30 & 65 & 100 & 365 \\
\hline F1 $(\mathrm{n}=5)$ & $100 \pm 0.0$ & $101.4 \pm 1.1$ & $102.0 \pm 2.3$ & $101.1 \pm 1.5$ & $97.2 \pm 2.1$ & $96.9 \pm 1.6$ & $96.7 \pm 1.7$ & $100.1 \pm 2.3$ \\
F2 $(\mathrm{n}=4)$ & $100 \pm 0.0$ & $103.8 \pm 0.9$ & $102.8 \pm 1.5$ & $100.8 \pm 2.2$ & $98.6 \pm 2.6$ & $98.4 \pm 1.1$ & $101.7 \pm 1.9$ & $101.5 \pm 2.1$ \\
\hline
\end{tabular}


protect the very damaged skin of these patients, thanks to an anti-radical action. ${ }^{20}$ Urea is traditionally used in dermatology for its moisturising and keratolytic action, ${ }^{21}$ which can be beneficial for patients with dry skin and hyperkeratosis. However, even if the low concentration used in gels is not sufficient to allow a keratolytic effect, urea can still act as an emollient. Our formulation meets both the objective of treating angiofibroma, as all studies published to date, and improves skin tolerance by adding the excipients mentioned above.

Finally, carbopol was added to our formulation because of its excellent tolerance and drug delivery capability. In addition, carbopol gels containing penetration activators such as ethanol have proven to be very effective and relatively easy to prepare.

No antimicrobial agents have been added due to the presence of ethanol and the bacteriostatic and antifungal properties of rapamycin. $^{22}$

The gel formulation offers better application characteristics than cream and ointment. Moreover, as Tanaka et al have demonstrated, the gel allows greater skin penetration than ointment according to in vitro tests and low irritation. ${ }^{23}$ Finally, the hydrophilic character induces a better aesthetic comfort for the patient and a better compliance.

In a recent study, Wataya-Kaneda et al concluded that the optimal concentration of rapamycin hydrophilic gel was $0.2 \%$, but observed more adverse events in the $0.2 \%$ rapamycin subgroup with dry skin (in $62 \%$ of cases) and irritation (in $50 \%$ of cases). In addition, low blood levels of rapamycin were detected in several patients, particularly in the paediatric subgroup $(50 \%$ and $100 \%$ in the adult subgroup and paediatric subgroup respectively), resulting in the likely occurrence of systemic side effects. ${ }^{24}$ In this context, our study proposed that $0.1 \%$ should appear as the highest concentration of rapamycin to achieve an excellent therapeutic index without any systemic side effects for the patient and at a lower cost.

Finally, the long stability obtained with our formulation, allows optimal patient management given the frequency of consultations (every 6 months minimum) and the frequent use of treatment beyond this consultation period.

\section{CONCLUSION}

This study describes a new gel formulation that is stable for 1 year, which could be effective against angiofibromas and safe in adulthood. Rapamycin has been solubilised in ethanol to improve skin penetration and various excipients have been added to promote tolerance. In addition, the hydrophilic nature of the formulation induces aesthetic comfort for the patient.

\section{What this paper adds}

What is already known on this subject

- Topical rapamycin is an effective and safe medical alternative to ablative treatments in patients with TSC-associated facial angiofibromas

- No curative treatment has been marketed in Europe

- Only one study published stability data on a cream but stability has only been achieved over 85 days

What this study adds

- This work would be the first to have studied a long-term physico-chemical stability of a new hydrophilic rapamycin gel with detailed data (1 year)

- This is the first study to propose the use of various excipients in order to improve skin penetration and skin tolerance
Acknowledgements The authors acknowledge Vincent Boudy and Benoit d'Hayer for their help during the rheological measurements and Bernard Do for his language assistance.

Funding The authors have not declared a specific grant for this research from any funding agency in the public, commercial or not-for-profit sectors.

Competing interests None declared.

Patient consent Not required.

Provenance and peer review Not commissioned; externally peer reviewed.

\section{REFERENCES}

1 Crall C, Valle M, Kapur K, et al. Effect of angiofibromas on quality of life and access to care in tuberous sclerosis patients and their caregivers. Pediatr Dermatol 2016;33:518-25

2 Li S, Takeuchi F, Wang JA, et al. Mesenchymal-epithelial interactions involving epiregulin in tuberous sclerosis complex hamartomas. Proc Natl Acad Sci U S A 2008; 105:3539-44.

3 Darling TN. Hamartomas and tubers from defects in hamartin-tuberin. J Am Acad Dermato/ 2004;51:9-11.

4 Koenig MK, Hebert AA, Roberson J, et al. Topical rapamycin therapy to alleviate the cutaneous manifestations of tuberous sclerosis complex. Drugs $R$ D 2012;12:121-6.

5 Salido-Vallejo R, Garnacho-Saucedo G, c M-GJ. Revisión: opciones terapéuticas actuales para los angiofibromas faciales. Curr Options Treat Facial Angiofibromas Engl 2014;105:558-68.

6 DeKlotz CM, Ogram AE, Singh S, et al. Dramatic improvement of facial angiofibromas in tuberous sclerosis with topical rapamycin: optimizing a treatment protocol. Arch Dermatol 2011;147:1117-7

7 Salido R, Garnacho-Saucedo G, Cuevas-Asencio I, et al. Sustained clinical effectiveness and favorable safety profile of topical sirolimus for tuberous sclerosis - associated facial angiofibroma. J Eur Acad Dermatol Venereol 2012;26:1315-8.

8 Tu J, Foster RS, Bint LJ, et al. Topical rapamycin for angiofibromas in paediatric patients with tuberous sclerosis: follow up of a pilot study and promising future directions. Australas J Dermatol 2014;55:63-9.

9 Lee $\mathrm{YI}$, Lee JH, Kim DY, et al. Comparative effects of topical $0.2 \%$ sirolimus for angiofibromas in adults and pediatric patients with tuberous sclerosis complex. Dermatology 2018;234:13-22.

10 Bouguéon G, Lagarce F, Martin L, et al. Formulation and characterization of a $0.1 \%$ rapamycin cream for the treatment of tuberous sclerosis complex-related angiofibromas. Int J Pharm 2016;509:279-84.

11 Il'ichev YV, Alquier L, Maryanoff CA. Degradation of rapamycin and its ring-opened isomer: role of base catalysis. ARKIVOC 2007:110-31.

12 Mutizwa MM, Berk DR, Anadkat MJ. Treatment of facial angiofibromas with topical application of oral rapamycin solution (1mgmL(-1)) in two patients with tuberous sclerosis. Br J Dermatol 2011:165:922-3.

13 Wheless JW, Almoazen H. A novel topical rapamycin cream for the treatment of facial angiofibromas in tuberous sclerosis complex. J Child Neurol 2013;28:933-6.

14 Haemel AK, O'Brian AL, Teng JM. Topical rapamycin: a novel approach to facial angiofibromas in tuberous sclerosis. Arch Dermatol 2010;146:715-8.

15 Malissen N, Vergely L, Simon M, et al. Long-term treatment of cutaneous manifestations of tuberous sclerosis complex with topical 1\% sirolimus cream: a prospective study of 25 patients. J Am Acad Dermatol 2017:77:464-72.

16 Foster RS, Bint LJ, Halbert AR. Topical $0.1 \%$ rapamycin for angiofibromas in paediatric patients with tuberous sclerosis: a pilot study of four patients. Australas I Dermatol 2012;53:52-6

17 Williams AC, Barry BW. Penetration enhancers. Adv Drug Deliv Rev 2004;56:603-18.

18 Hara M, Verkman AS. Glycerol replacement corrects defective skin hydration, elasticity, and barrier function in aquaporin-3-deficient mice. Proc Natl Acad Sci U S A 2003;100:7360-5.

19 Fluhr JW, Darlenski R, Surber C. Glycerol and the skin: holistic approach to its origin and functions. Br J Dermatol 2008;159:23-34.

20 Nachbar F, Korting HC. The role of vitamin E in normal and damaged skin. J Mol Med 1995:73:7-17.

21 Friedman AJ, von Grote EC, Meckfessel MH. Urea: A clinically oriented overview from bench to bedside. J Drugs Dermatol 2016;15:633-9.

22 Vézina C, Kudelski A, Sehgal SN. Rapamycin (AY-22,989), a new antifungal antibiotic. I. Taxonomy of the producing streptomycete and isolation of the active principle. $J$ Antibiot 1975;28:721-6.

23 Tanaka M, Wataya-Kaneda M, Nakamura A, et al. First left-right comparative study of topical rapamycin vs. vehicle for facial angiofibromas in patients with tuberous sclerosis complex. Br J Dermatol 2013:169:1314-8.

24 Wataya-Kaneda M, Nakamura A, Tanaka M, et al. Efficacy and safety of topical sirolimus therapy for facial angiofibromas in the tuberous sclerosis complex: a randomized clinical trial. JAMA Dermatol 2017;153:39-48. 